\title{
Investigative Study of Planar Array Ambiguities Based on "Hyperhelical" Parameterization
}

\author{
Athanassios Manikas, Member, IEEE, Christos Proukakis, and Vasileios Lefkaditis
}

\begin{abstract}
In this paper, a novel approach is proposed for the calculation of ambiguous generator sets of directions for planar arrays. This approach is based on the concept of hyperhelical curves and their equivalent linear arrays, which provide a vehicle for investigating planar array ambiguities by adopting and extending techniques proposed for linear arrays. Thus, ambiguous generator sets of directions of constant azimuth and different elevation are initially calculated, and the concept of ambiguous generator lines is introduced. Then, by using coneangle parametrization, ambiguities associated with directions of different azimuth and different elevation are investigated.
\end{abstract}

Index Terms - Ambiguities, array processing, differential geometry, planar arrays.

\section{INTRODUCTION}

$\mathbf{I}^{\mathrm{s}}$ $\mathrm{N}$ AN azimuth-elevation direction finding (DF) system employing a planar array of $N$ isotropic sensors, the array manifold is the locus of the response vectors (or, manifold vectors) $\underline{\mathrm{a}}(\theta, \phi) \forall \theta, \phi$

$$
\begin{aligned}
\underline{\mathrm{a}}(\theta, \phi) & =\exp (-j \underline{\underline{\mathbf{r}}} \underline{k}(\theta, \phi)) \\
& =\exp \left(-j \pi\left(\underline{r}_{x} \cos \theta+\underline{r}_{y} \sin \theta\right) \cos \phi\right)
\end{aligned}
$$

and has the shape of a conoid lying on a hypersphere of radius $\sqrt{N}$ in the complex $N$-dimensional space $\mathcal{C}^{N}$ [1].

In (1), $\underline{\underline{\mathbf{r}}}=\left[\underline{r}_{x}, \underline{r}_{y}, 0\right] \in \Re^{N \times 3}$ denotes the array sensor locations in half wavelengths, and $\underline{k}(\theta, \phi)=$ $\pi[\cos \phi \cos \theta, \cos \phi \sin \theta, \sin \phi]^{T} \in \Re^{3 \times 1}$ is the wavenumber vector pointing toward the emitter at azimuth $\theta$ and elevation $\phi$ [with $\theta$ measured anticlockwise from the $x$-axis on the $x-y$ plane $\theta \in[0,2 \pi), \phi \in[0, \pi / 2)]$.

The geometry of the array elements plays a crucial role in dictating the shape, properties, and "anomalies" of the array manifold and, as a consequence, in dictating the phenomenon where some manifold vectors can be written as a linear combination of some other manifold vectors. Note that in [2], a necessary condition to obtain unique estimates of directionsof-arrival (DOA's) was proposed based on the assumption of linearly independent manifold vectors. This condition is a function of the number of sensors, the number of sources, and the rank of the source correlation matrix and was improved in [3], whereas in [4] and [5], it was extended to vector-sensor applications.

Manuscript received July 7, 1997; revised November 27, 1998. The associate editor coordinating the review of this paper and approving it for publication was Prof. Chi Chung Ko.

The authors are with the Communications and Signal Processing Research Section, Department of Electrical and Electronic Engineering, Imperial College of Science, Technology, and Medicine, London, U.K.

Publisher Item Identifier S 1053-587X(99)03639-9.
However, if some of the manifold vectors are linearly dependent, then the ambiguity problem is said to arise, implying a need to identify array geometries that are free of some type of ambiguities, as well as estimating the set of ambiguous directions associated with a given geometry, which are both very important problems in direction finding.

The ambiguities are characterized as trivial, which are easy to identify, or as nontrivial, with the nontrivial ambiguities being the most challenging and, therefore, interesting type of ambiguities. Thus, much research effort has been devoted to the question of identifying specific array geometries that are free of nontrivial ambiguities, up to a certain rank, or studying the performance of certain array geometries. In [6], a class of cross arrays is constructed that is free of higher rank ambiguities, and in [7], a theorem for characterizing rank2 ambiguities is derived. In [8], a specific class of uniform circular arrays is shown to be free of rank-2 ambiguities when the sources are coplanar with the array. As far as the estimation of ambiguities is concerned, in [9] and [10], linear arrays were studied, based on uniform and nonuniform partitioning of the array manifold, and thus, two classes of ambiguous generator sets of directions were estimated. However, the authors have no knowledge of any attempt to estimate ambiguities associated with planar array structures.

In this paper, the ambiguities of planar arrays of any geometry are investigated and estimated for the case of directions of constant azimuth and different elevations as well as for the case of directions of different azimuths and different elevations. However, the case of estimating ambiguous sets of different azimuth and constant elevations (if there are any) is not examined in this study and remains an open problem.

In Section II, a background framework on the properties of the manifold of a linear array is given together with a brief presentation of the basic ambiguity theory concerning their estimation in linear arrays. In Section III, the concept of the equivalent linear array (ELA) is presented, and the family of hyperhelical curves, known as $\phi$-curves, is considered in order to obtain ambiguous sets of directions of constant azimuth and different elevation. The methodology of calculating the locus of ambiguous generator sets of a planar array is then highlighted through a representative example, and thus, the concept of ambiguous generator lines is introduced. In Section IV, based on the cone angle parametrization of the array manifold, two additional families of hyperhelical curves ( $\alpha$ and $\beta$-curves), which can be used to estimate ambiguous sets of directions of different azimuth and different elevation, are presented. Then, a number of observations are made that 
place these three families of hyperhelical curves $(\phi, \alpha$, and $\beta$-curves) in a general framework. Finally, in Section V, some special cases of low-rank ambiguities are discussed, and in Section VI, the paper is concluded.

\section{BACKGROUND THEORY}

The influence of the array manifold on the performance of a DF system can be investigated using differential geometry of curves and surfaces [11], [12].

For instance, if $p$ is the parameter of interest, then the function

$$
\underline{\mathrm{a}}(p)=\exp (-j \pi(\underline{r} \cos p+\underline{n})) \quad \forall p \in \Omega_{p}
$$

describes a curve in the complex $N$-dimensional space and represents the locus of all vectors $\mathrm{a}(p)$ over the parameter space $\Omega_{p}$, where $\underline{r}, \underline{n}$ are constant $N$-dimensional real vectors.

Curves of the form of (2) have a hyperhelical shape, and the advantages of having hyperhelical curves are numerous. The most important is that their shape and properties can be described uniquely by a set of constant curvatures. Those curvatures, which do not vary from point to point (i.e., are independent of $p$ ), can be estimated analytically [13], [14] and form the Cartan matrix, which is a $d \times d$ skew-symmetric matrix defined as

$$
\mathbb{C} \stackrel{\text { def }}{=}\left[\begin{array}{cccccc}
0 & -\kappa_{1} & 0 & 0 & \cdots & 0 \\
\kappa_{1} & 0 & -\kappa_{2} & 0 & \cdots & 0 \\
0 & \kappa_{2} & 0 & -\kappa_{3} & \cdots & 0 \\
\vdots & \vdots & \vdots & \vdots & \vdots & \vdots \\
0 & 0 & \cdots & \kappa_{d-2} & 0 & -\kappa_{d-1} \\
0 & 0 & \cdots & 0 & \kappa_{d-1} & 0
\end{array}\right]
$$

where

$$
\kappa_{i}=i \text { th curvature }
$$

and where

$$
d=\left\{\begin{array}{l}
2 N-m \\
\quad \text { if no element of vector } \underline{r} \text { is at the centroid of } \underline{r} \\
2 N-m-1 \\
\text { otherwise }
\end{array}\right.
$$

with $m$ representing the number of elements of $\underline{r}$ that have symmetrical pairs with respect to its centroid.

The most basic parameter of a curve, however, is the arc length $s$, which is defined as

$$
s(p) \stackrel{\text { def }}{=} \int_{0}^{p}\left\|\frac{\mathrm{da}(p)}{\mathrm{d} p}\right\| \mathrm{d} p
$$

and, if the curve is hyperhelix, is related to the parameter $p$ (with $s=0$ at $p=0$ ) as

$$
p(s)=\cos ^{-1}\left(1-\frac{s}{\pi\|\underline{r}\|}\right)
$$

while the rate of change of arc length with respect to the parameter $p$ is given by

$$
\dot{s}(p)=\frac{\mathrm{d} s}{\mathrm{~d} p}=\|\underline{\mathrm{a}}(p)\|
$$

with () used to represent differentiation with respect to parameter $p$.
Finally, the total length of this complex curve is equal to

$$
l_{m}=s(\pi)=2 \pi\|\underline{x}\|
$$

The above parameters and the concept of a hyperhelical curve have been employed in [9] to handle the ambiguity problem of linear arrays with sensor locations $\underline{r} \in R^{N}$ (with $\underline{n}$ an all zeros vector), and the following definition of an ambiguous set and its associated rank of ambiguity was presented.

Ambiguous Set: An ordered set of arc lengths $\underline{s}=$ $\left[s_{1}, s_{2}, \cdots, s_{m}\right]^{T}$, where $m \leq N$, is said to be an ambiguous set of arc lengths if the matrix $\mathrm{A}(\underline{s}) \in \mathcal{C}^{N \times m}$ with columns the manifold vectors $\underline{\mathrm{a}}\left(s_{1}\right), \underline{\mathrm{a}}\left(s_{2}\right), \cdots, \underline{\mathrm{a}}\left(s_{m}\right)$ has rank less than $m$, i.e., $\operatorname{rank}(A(\underline{s}))<m$.

Rank of Ambiguity: If a set of arc lengths $\underline{s}$ is ambiguous, then its rank of ambiguity is defined as the integer $\rho_{\mathbf{a}}=$ $\operatorname{rank}(\mathcal{A}(\underline{s}))$.

However, [9, Th. 1] essentially states that if all the elements of an ambiguous set of arc lengths are rotated on the array manifold by the same value, then the resulting set is also an ambiguous set of arc lengths. It becomes clear that if one ambiguous set is identified, then by simple rotation, an infinite number of ambiguous sets can be generated, and therefore, two different ambiguous sets may in fact be just a rotation of each other. Thus, since all these sets can be generated from a single set, the idea of the ambiguous generator set was proposed in [9] and is presented in the following.

Ambiguous Generator Set: An ordered set $\underline{s}=$ $\left[0, \mathrm{~s}_{1}, \cdots, s_{m-1}\right]^{T}$ of $m$ arc lengths, where $2 \leq m \leq N$, is said to be an ambiguous generator set of arc lengths if and only if we have the following.

a) All the elements of the set but the first element are nonzero.

b) The rank of the $N \times m$ matrix $A(\underline{s})$ with columns the manifold vectors associated with the elements of the set is less than $m$, i.e., $\operatorname{rank}((\mathbb{A}(\underline{s}))=r<m$.

c) For any subset $\underline{s}_{i}$ of $k$ elements of $\underline{s}$ with $r \leq k<m$, the rank of $\mathrm{A}\left(\underline{s}_{i}\right)$ is equal to $r$.

Based on the "hyperhelical" properties of the manifold of a linear array, two classes of ambiguous generator sets were identified. The first class is generated by uniform partitions of the array manifold based on the elements of the vector

$$
\underline{s}_{i, j}=\left[0, \frac{l_{m}}{\left|r_{i}-r_{j}\right|}, 2 \frac{l_{m}}{\left|r_{i}-r_{j}\right|}, \cdots, c \frac{l_{m}}{\left|r_{i}-r_{j}\right|}\right]^{T}
$$

where $r_{i}, r_{j}$ are the locations of the $i$ th and $j$ th sensors, respectively. Although this first class of ambiguity exists in any linear array geometry (symmetric or nonsymmetric), the second class of ambiguity can be found only in symmetric arrays and can be estimated by using a nonuniform partitioning of the array manifold based on the roots (which are smaller than the manifold length) of

$$
\operatorname{Tr}(\mathbb{C} \operatorname{expm}\{s \mathbb{C}\})=0
$$



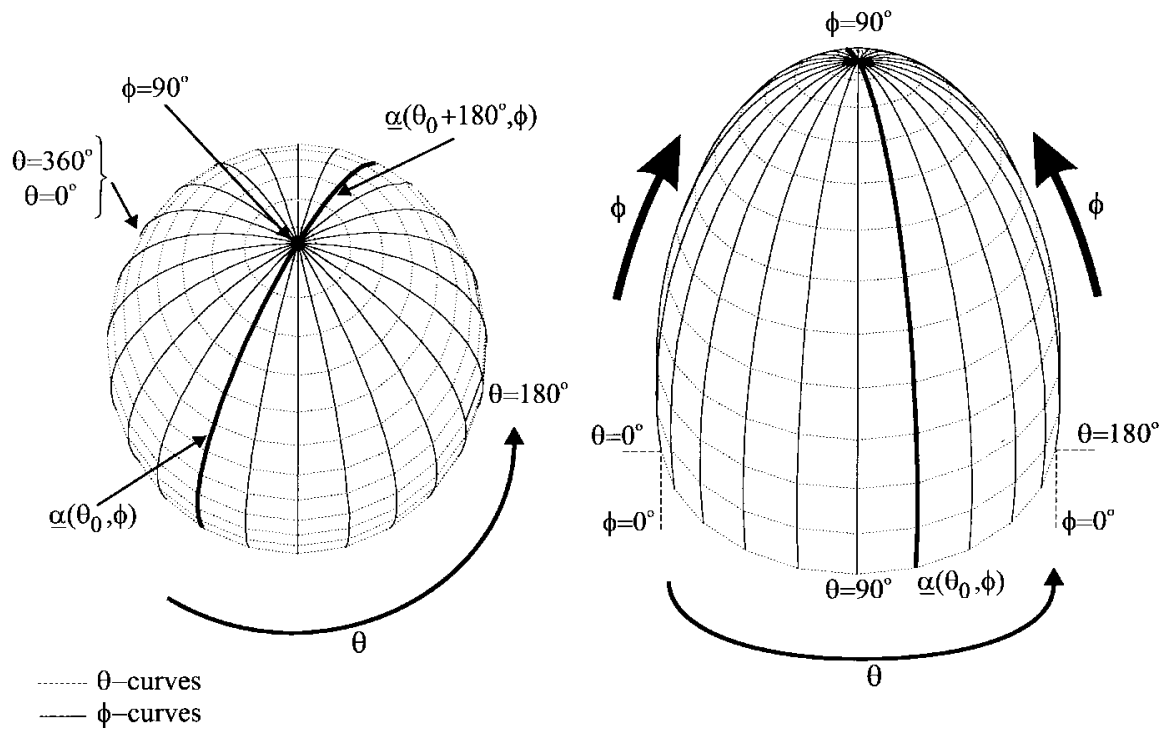

Fig. 1. Array manifold and its families of $\theta$ - and $\phi$-curves.

where

$$
\left\{\begin{array}{l}
s=\pi\|\underline{x}\|(1-\cos \theta) \\
\operatorname{expm}\{\cdot\} \text { denotes matrix exponential. }
\end{array}\right.
$$

There is a direct connection between the presence of grating lobes in the array pattern and the ambiguities associated with the roots of (9). It can be proved that if the array pattern is parametrized in terms of the arc length $s$ and the mainlobe of the array is steered toward endfire, then for a symmetric array of $N$ sensors, the $N$ stationary points (usually lobes) of the array pattern correspond to the ambiguous directions with a rank of ambiguity equal to $N-1$.

In the following sections, the array manifold surface of planar arrays is investigated by considering this surface as a family of properly parametrized curves that can be analyzed by adopting and extending the above concepts.

\section{Ambiguities Associated WITH THE FAMILY OF $\phi$-CURVES}

As it was mentioned in Section I, the shape of the manifold of a planar array is a conoid surface lying on a hypersphere of radius $\sqrt{N}$ in the complex $N$-dimensional space $\mathcal{C}^{N}$. This conoid surface is very difficult to analyze as a single entity. For this reason, an alternative mapping, representing this surface, is needed in order to investigate the properties of the manifold of planar arrays.

An alternative mapping is produced by treating the manifold surface as a family of curves that fully covers and describes the corresponding surface. Two such families of curves are

$\{$ the family of $\theta$-parameter curves (or simply $\theta$-curves) $\{$ the family of $\phi$-parameter curves (or simply $\phi$-curves)

with a curve belonging to the family of $\theta$-curves defined as the locus of all manifold vectors over the whole azimuth space $\Omega_{\theta} \equiv\left[0-360^{\circ}\right)$ at a particular elevation $\phi_{0}$

$$
\text { i.e., } \underline{\mathrm{a}}\left(\theta \mid \phi_{0}\right)=\left.\underline{\mathrm{a}}(\theta, \phi)\right|_{\forall \theta} ^{\phi=\phi_{0}} .
$$

On the other hand, a curve belongs to the family of $\phi$-curves if it is the locus of all manifold vectors formed by keeping the azimuth $\theta$ constant (with value $\theta_{0}$ ) and by varying the elevation over the whole elevation space $\Omega_{\phi}=\left[0-90^{\circ}\right)$

$$
\text { i.e., } \underline{\mathrm{a}}\left(\phi \mid \theta_{0}\right)=\left.\underline{\mathrm{a}}(\theta, \phi)\right|_{\forall \phi} ^{\theta=\theta_{0}} \text {. }
$$

Both families of curves can be used to describe the manifold surface, but the $\theta$-parameter curves are the most complex of the two families. The $\phi$-curves (in contrast to the $\theta$-curves) have the property of being complex hyperhelices embedded in $C^{N}$. This can be seen from (1) for constant $\theta$ (of value $\theta_{0}$ )

$$
\begin{aligned}
\underline{\mathrm{a}}\left(\theta_{0}, \phi\right) & =\exp \left(-j \pi\left(\underline{r}_{x} \cos \theta_{0}+\underline{r}_{y} \sin \theta_{0}\right) \cos \phi\right) \\
\text { i.e., } \underline{\mathrm{a}}\left(\theta_{0}, \phi\right) & =\exp \left(-j \pi \underline{R}\left(\theta_{0}\right) \cos \phi\right)
\end{aligned}
$$

where

$$
\underline{R}\left(\theta_{0}\right)=\underline{r}_{x} \cos \theta_{0}+\underline{r}_{y} \sin \theta_{0}
$$

which matches (2) for $\underline{r}=\underline{R}\left(\theta_{0}\right), p=\phi$, and $\underline{n}=\underline{0}$.

Note that hyperhelical curves, such as $\phi$-curves, are analytically "convenient" in the sense that all their curvatures are independent of the parameter $p$, and hence, the procedure for their calculation is identical to that of linear arrays [9]. Fig. 1 shows an illustrative representation of a planar array manifold surface and its families of $\theta$ - and $\phi$-curves.

It is immediately apparent that

$$
\underline{\mathrm{a}}\left(\theta_{0}, \phi\right)=\underline{\mathrm{a}}\left(\theta_{0}+180^{\circ}, \phi\right)
$$

which implies that the two $\phi$-curves at $\theta=\theta_{0}$ and $\theta=$ $\theta_{0}+180^{\circ}$ have the same length and curvatures and can be considered as a continuation of one another forming a composite $\phi$-curve having a hyperhelical shape. The family of these composite curves (hereafter known as $\phi_{c}$-curves) can be described in an alternative but equivalent parameter space as $\left\{\left(\hat{\theta}_{0}, \hat{\phi}\right): \hat{\theta}_{0} \in\left[0^{\circ}, 180^{\circ}\right), \hat{\phi} \in\left[0^{\circ}, 180^{\circ}\right)\right\}$, i.e., $\Omega_{\hat{\theta}}=\Omega_{\hat{\phi}}=\left[0-180^{\circ}\right)$, which is connected to the original 
space of the family of the $\phi$-curves as

$$
\begin{aligned}
& \text { if } \hat{\phi} \geq 90^{\circ} \text { then }\left\{\begin{array}{l}
\phi=180^{\circ}-\hat{\phi} \\
\theta_{0}=\hat{\theta}_{0}+180^{\circ}
\end{array}\right. \\
& \text { if } \hat{\phi}<90^{\circ} \text { then }\left\{\begin{array}{l}
\phi=\widehat{\phi} \\
\theta_{0}=\hat{\theta}_{0} .
\end{array}\right.
\end{aligned}
$$

It is obvious now that a $\phi_{c}$-curve represents directly the array manifold of an ELA with sensor locations given by $\underline{R}\left(\hat{\theta}_{0}\right)$ having a manifold length

$$
l_{m_{\mathrm{FLA}}}\left(\hat{\theta}_{0}\right)=2 \pi\left\|\underline{R}\left(\hat{\theta}_{0}\right)\right\| .
$$

If $r_{i}, r_{j}$ denote the locations of the $i$ th and $j$ th sensors of the ELA $\underline{R}\left(\hat{\theta}_{0}\right)$, then the ambiguous generator sets of arc lengths can be estimated by partitioning the manifold of this ELA according to the elements of the vector [see (8)]

$$
\begin{gathered}
s_{i, j}=\left[0, \frac{l_{m_{\mathrm{FLA}}}}{\left|r_{i}-r_{j}\right|}, 2 \frac{l_{m_{\mathrm{FLA}}}}{\left|r_{i}-r_{j}\right|}, \cdots, c \frac{l_{m_{\mathrm{FLA}}}}{\left|r_{i}-r_{j}\right|}\right]^{T} \\
\forall i, j \text { with } i \neq j
\end{gathered}
$$

with the composite elevations $\hat{\phi}_{k}$, corresponding to the arc lengths $s_{k}$ of the $\phi_{c}$-curve, calculated by

$$
\hat{\phi}_{k}=\cos ^{-1}\left(1-\frac{s_{k}}{\pi\left\|R\left(\hat{\theta}_{0}\right)\right\|}\right) .
$$

For instance, for the following planar array of six sensors (in half-wavelengths)

$$
\left[\underline{r}_{x}, \underline{r}_{y}, 0\right]=\left[\begin{array}{rrrrrr}
-2.5, & -2, & -2.5, & 2.5, & 2, & 2.5 \\
1.5, & 0, & -1.5, & 1.5, & 0, & -1.5 \\
0, & 0, & 0, & 0, & 0, & 0
\end{array}\right]^{T}
$$

the ELA associated with, say, $\hat{\theta}_{0}=5^{\circ}$, is $\underline{R}\left(\hat{\theta}_{0}\right)=$ $[-2.6212,-2.3598,-1.9924,1.9924,2.3598,2.6212]^{T}$ having a manifold length equal to $l_{m_{\mathrm{ELA}}}\left(\hat{\theta}_{0}\right)=35.9943$. By using (17) in conjunction with the technique proposed in [9], the following four ambiguous generator sets of arc lengths are obtained as in (20), shown at the bottom of the page.

The first ambiguous generator set (first row of $\underline{\underline{\underline{\Sigma}}}$ ) for this specific ELA of $\hat{\theta}_{0}=5^{\circ}$ has rank of ambiguity equal to 5 , and its nonzero elements are shown as dots in Fig. 2. Note that the directions in degrees associated with the above ambiguous generator set of arc lengths are $\left[\left(5^{\circ}, 0^{\circ}\right),\left(5^{\circ}, 51.79^{\circ}\right),\left(5^{\circ}, 76.29^{\circ}\right),\left(185^{\circ}, 81.69^{\circ}\right)\right.$, $\left.\left(185^{\circ}, 58.26^{\circ}\right),\left(185^{\circ}, 24.84^{\circ}\right)\right]$.

Furthermore, in the same figure, the locus of the manifold lengths of all ELA's, $l_{m_{\mathrm{ELA}}}\left(\hat{\theta}_{0}\right)$, is shown, i.e., $l_{m_{\mathrm{FLA}}}\left(\hat{\theta}_{0}\right), \forall \hat{\theta}_{0}$.

However, the points of an ambiguous generator set, like the one shown in Fig. 2, belong to a set of ambiguous generator lines with each line of the set representing the locus of one of

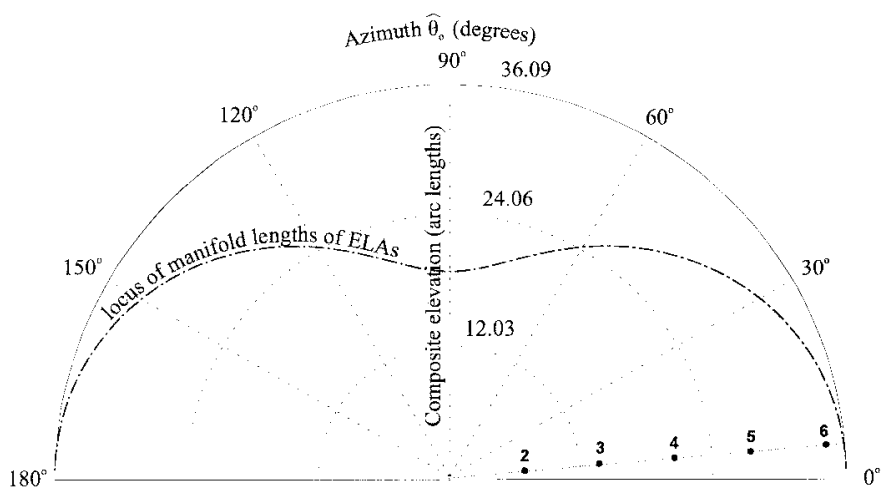

Fig. 2. Ambiguous generator set corresponding to the first row of $\supseteqq$.

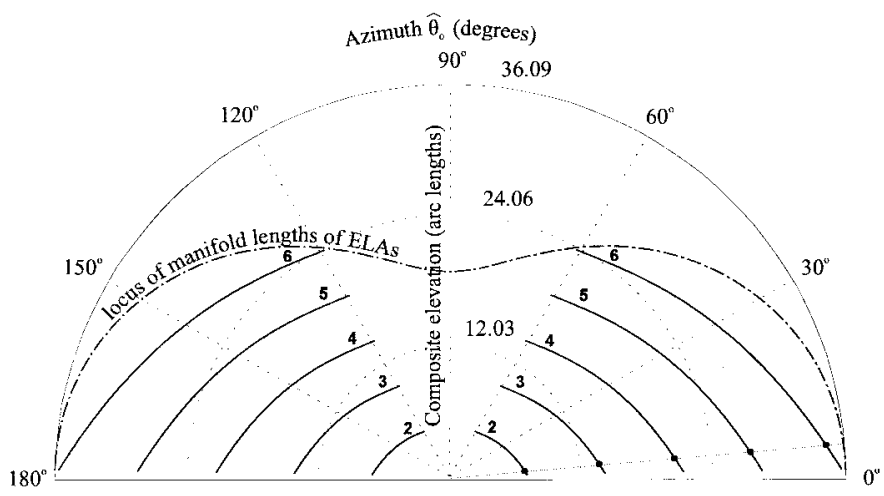

Fig. 3. Set of ambiguous generator lines of rank-5 in which the elements of the first row of $\equiv$ belong.

the elements of this generator set for every $\hat{\theta}_{0}$ in $\Omega_{\hat{\theta}}$. Thus, Fig. 3 shows the set of ambiguous generator lines of rank 5 in which the first row of $\underline{\underline{\underline{\Sigma}}}$ belongs. As can be seen in this figure, the set of ambiguous generator lines ceases to exist at $\hat{\theta}_{0}=0$ and for values of $\hat{\theta}_{0}$ in region $61-119^{\circ}$, whereas for $\hat{\theta}_{0}$ in region $119-180^{\circ}$, its values are the mirror image of the values from $0-61^{\circ}$. Note that the set of ambiguous generator lines is defined only in those areas of the parameter space $\Omega_{\hat{\theta}}$ at which the last line (e.g., in Fig. 3 the sixth line) where the largest element of the associated ambiguous generator set is located is below the locus of the manifold length of the ELA's. In addition, in Figs. 4-6, the sets of ambiguous generator lines in which rows $2-4$ of $\sum$ belong are shown. Note that in Fig. 4, in addition to the set of ambiguous generator lines of rank-4, which is shown by the set of solid black lines and which do not exist at $\hat{\theta}_{0}=45^{\circ}$, at $\hat{\theta}_{0}=135^{\circ}$, and for $\hat{\theta}_{0}$ in region $120-180^{\circ}$, two extra "discrete" ambiguous generator sets of rank 2 and one of rank 1 are shown as black squares, which appear at $\hat{\theta}_{0}=45^{\circ}, \hat{\theta}_{0}=135^{\circ}$, and $\hat{\theta}_{0}=90^{\circ}$ having the following values:

$$
\begin{aligned}
& \hat{\theta}_{0}=45^{\circ}:[0,13.5732,27.1465], \quad \rho=2 \\
& \hat{\theta}_{0}=135^{\circ}:[0,13.5732,27.1465], \quad \rho=2 \\
& \hat{\theta}_{0}=90^{\circ}:[0,12.5664], \quad \rho=1 .
\end{aligned}
$$

$$
\underline{\underline{\Sigma}}=\left[\begin{array}{cccccc}
0, & 6.8659, & 13.7319, & 20.5978, & 27.4638, & 34.3297 \\
0, & 7.2264, & 14.4527, & 21.6791, & 28.9054, & 0 \\
0, & 7.8018, & 15.6035, & 23.4053, & 31.2071, & 0 \\
0, & 8.2705, & 16.5410, & 24.8114, & 33.0819, & 0
\end{array}\right] \quad \rho_{1 \alpha}=\left[\begin{array}{c}
5 \\
4 \\
4 \\
4
\end{array}\right]
$$




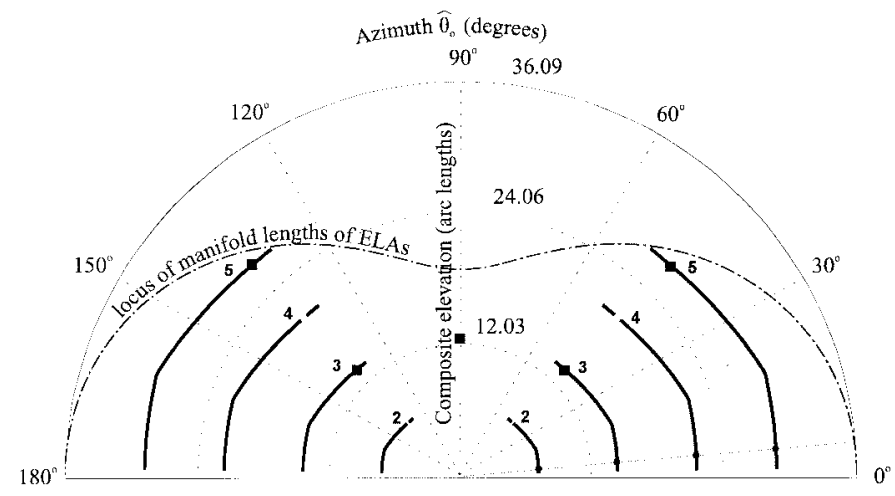

Fig. 4. Set of ambiguous generator lines of rank-4 in which the elements of the second row of $\Sigma$ belong. The figure also shows two generator sets of rank-2 and one of rank-1 (trivial ambiguity) as black squares.

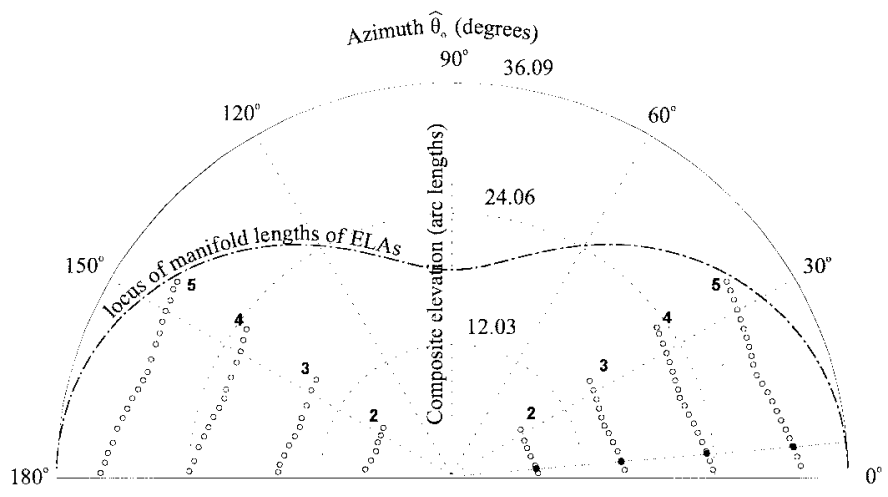

Fig. 5. Set of ambiguous generator lines of rank-4 in which the elements of

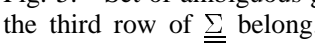

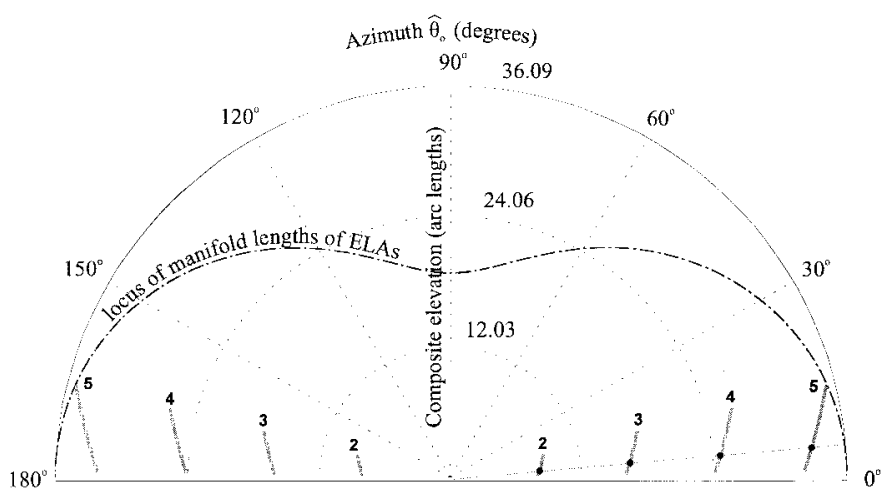

Fig. 6. Set of ambiguous generator lines of rank-4 in which the elements of the fourth row of $\underline{\equiv}$ belong.

These "discrete" ambiguous generator sets will be discussed in a later section.

Thus far, ambiguous generator sets based on uniform partitioning of a hyperhelical curve and their associated set of ambiguous generator lines have been discussed. However, it is worth noting that ELA's of some curves of the family of $\phi_{c}$-curves of a planar array may be symmetric, i.e., $\operatorname{sum}\left(\underline{R}\left(\hat{\theta}_{0}\right)^{n}\right)=0, \forall n=$ odd. In this case, extra ambiguities can be found by a nonuniform partitioning of those $\phi_{c}$-curves according to the roots of (9), which are smaller than the manifold length of the ELA. Furthermore if the planar array itself is symmetric, then all ELA's of the family of $\phi_{c}$-curves

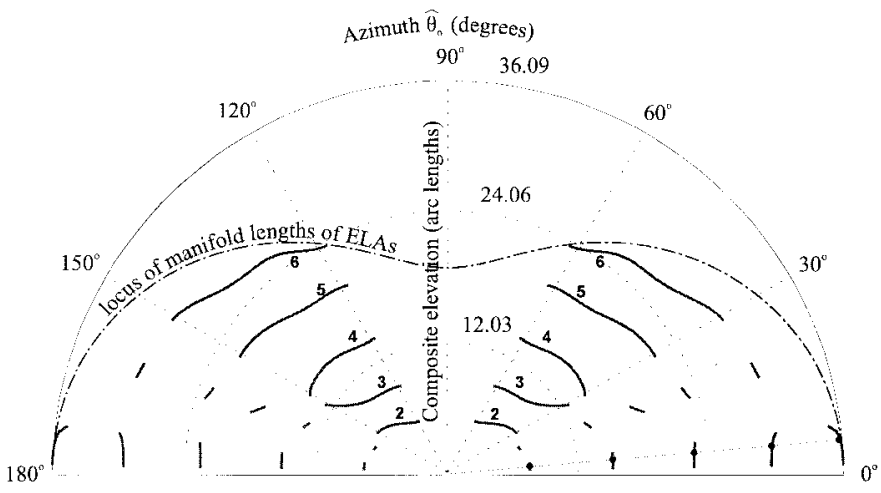

Fig. 7. Set of ambiguous generator lines of rank-5 in which the elements of the generator set of nonuniform partition belong.

are symmetric as well; this is formally stated in the following corollary.

Corollary: All equivalent linear arrays associated with the family of $\phi_{c}$-curves of a symmetric planar array are symmetric and, therefore, can suffer from both uniform and nonuniform classes of ambiguity.

For instance, the planar array of (19) is a symmetric array, and therefore, the nonuniform class of ambiguities may also be present. Thus, for example, for the ELA of $\hat{\theta}_{0}=5^{\circ}$, the roots of (9), which are smaller than the manifold length $l_{m_{\mathrm{ELA}}}\left(\hat{\theta}_{0}\right)=35.9943$, are

$$
\underline{s}=[0,7.6422,15.2410,22.7093,29.7888,35.8895]^{T}
$$

of rank-5.

This should be added as an extra row (fifth row) to the matrix $\underline{\underline{\Sigma}}$ of (20), and the corresponding set of ambiguous generator lines is shown in Fig. 7. In Fig. 8, the diagrams of Figs. 3-7 are superimposed, indicating that the array is free of $\phi$-curve ambiguities for any azimuth between $61-119^{\circ}$ with the only exception at $90^{\circ}$, where there exists a single "discrete" rank-1 ambiguous generator set, which is a trivial type of ambiguity. This is not the only type of trivial ambiguity that can be found since, like linear arrays, planar arrays are also sensitive to trivial ambiguities when the parameter space of the family of $\phi$-curves $\Omega_{\phi}$ increases from $\left[0-90^{\circ}\right.$ ) to [ -90 to $90^{\circ}$ ). This becomes apparent from (11) because

$$
\mathrm{a}\left(\theta_{0}, \phi\right)=\mathrm{a}\left(\theta_{0},-\phi\right)
$$

which implies that a planar array is incapable of distinguishing between two emitters of equal azimuth $\theta_{0}$ and opposite elevations $\pm \phi$, which is the reason why the elevation is restricted to $\left[0^{\circ}, 90^{\circ}\right)$ [or, the composite elevation is restricted to $\left[0^{\circ}\right.$, $\left.\left.180^{\circ}\right)\right]$.

\section{Ambiguities of $\alpha$ - AND $\beta$-CuRVE FAMILIES}

A different parametrization known as cone-angle parametrization may also be used for the study of the manifold. The new angles are known as the cone angles $\alpha, \beta$, where $\alpha$ is defined as the angle between $\underline{k}(\theta, \phi)$ and the $x$-axis, and $\beta$ is the angle between $\underline{k}(\theta, \phi)$ and the $y$-axis. Additionally, if the $x$ - and $y$-axes of the Cartesian frame $x-y-z$ are rotated by an angle $\Theta$, then the frame 


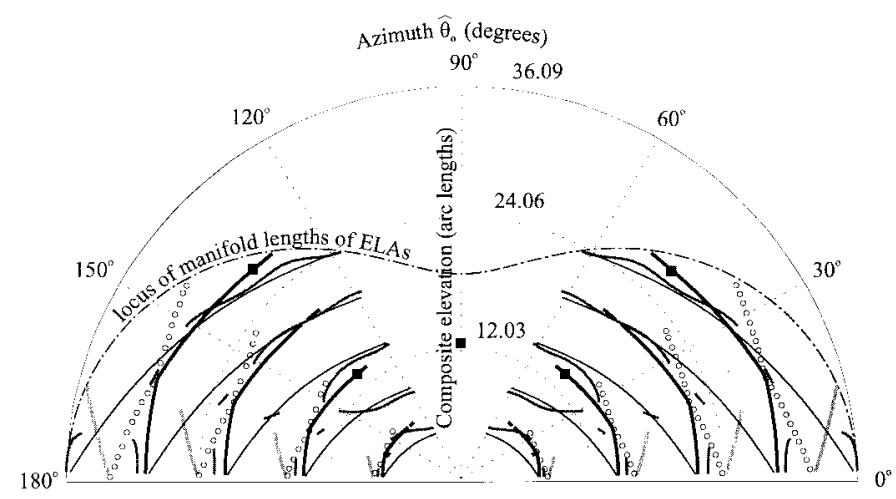

Fig. 8. All the sets of ambiguous generator lines for the $\phi_{c}$-curves of the planar array of (19).

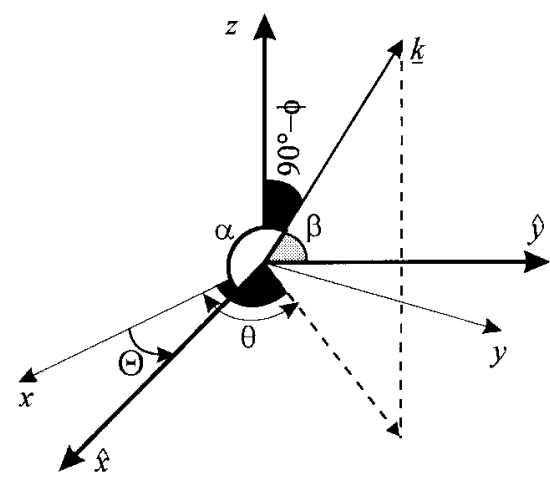

(a)

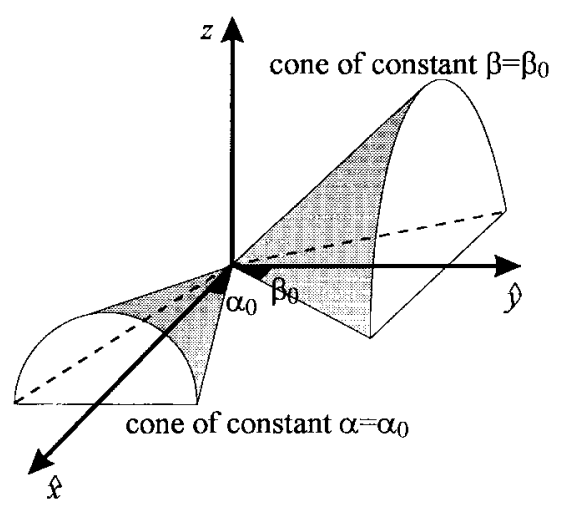

(b)

Fig. 9. (a) Azimuth-elevation and $\alpha-\beta$ conventions. (b) Cones formed for $\alpha=\alpha_{0}$ and $\beta=\beta_{0}$.

$\hat{x}-\hat{y}-z$ is obtained. In this case, $\alpha$ is defined as the angle between $\underline{k}(\theta, \phi)$ and the $\hat{x}$-axis, whereas $\beta$ is the angle between $\underline{k}(\theta, \phi)$ and the $\hat{y}$-axis. The relation between the two conventions is illustrated in Fig. 9(a). These angles are called cone angles because the loci of wavenumber vectors of constant $\alpha=\alpha_{0}$ (or equivalently constant $\beta=\beta_{0}$ ) form cones about the $\hat{x}$ (equivalently, the $\hat{y}$ ) axis. Fig. 9(b) shows this for a random choice of $\alpha_{0}$ and $\beta_{0}$.

The wavenumber vector with respect to the cone angles and for a rotation angle $\Theta$ can be written as

$$
\underline{\hat{k}}(\alpha, \beta)=\pi\left[\cos \alpha, \cos \beta, \sqrt{1-\cos ^{2} \alpha-\cos ^{2} \beta}\right]^{T}
$$

where

$$
\begin{array}{ll}
\cos \alpha=\cos \phi \cos (\theta-\Theta) & 0^{\circ} \leq \alpha \leq 180^{\circ} \\
\cos \beta=\cos \phi \sin (\theta-\Theta) & 0^{\circ} \leq \beta \leq 180^{\circ}
\end{array}
$$

and, thus, the manifold vector of a planar array can be expressed as a function of the $\alpha$ and $\beta$ parameters as

$$
\begin{aligned}
\underline{\hat{a}}(\alpha, \beta) & =\exp (-j \pi \underline{\underline{\hat{\mathbf{r}}}} \underline{\hat{k}}(\alpha, \beta)) \\
& =\exp \left(-j \pi\left(\underline{R}(\Theta) \cos \alpha+\underline{R}\left(\Theta+\frac{\pi}{2}\right) \cos \beta\right)\right)
\end{aligned}
$$

where $\underline{\underline{\hat{r}}}$ is the matrix of the sensor locations, which are given as

$$
\underline{\underline{\hat{r}}}=\left[\underline{R}(\Theta), \underline{R}\left(\Theta+\frac{\pi}{2}\right), 0\right]
$$

with

$$
\underline{R}(\Theta)=\underline{r}_{x} \cos \Theta+\underline{r}_{y} \sin \Theta .
$$

From the previous equation, it is easy to deduce that the roles of $\alpha$ and $\beta$ can be interchanged by simply replacing $\Theta$ with $\Theta+\pi / 2$.

The expressions that calculate the azimuth $\theta$ and the elevation $\phi$ for a given pair of values of cone angles $\alpha$ and $\beta$ and a given rotation $\Theta$ of a $x-y$ frame are derived from (23) and (24) and are

$$
\begin{aligned}
\theta & =\theta(\Theta)=\Theta+\tan ^{-1}\left(\frac{\cos \beta}{\cos \alpha}\right) \\
\phi & =\cos ^{-1}\left(\frac{\cos \beta}{\sin (\theta-\Theta)}\right) \\
& =\cos ^{-1}\left(\frac{\cos \beta}{\sin \left(\tan ^{-1}\left(\frac{\cos \beta}{\cos \alpha}\right)\right)}\right) .
\end{aligned}
$$

Note that when $\beta=90^{\circ}$, the above (28) and (29) are not valid. In this case, $\theta=\Theta$ or $\theta=\Theta+180^{\circ}$ and $\phi=\alpha$ or $\phi=180^{\circ}-\alpha$, depending on whether $\alpha \leq 90^{\circ}$ or $\alpha>90^{\circ}$.

From the above equations, it is obvious that unlike $\theta-\phi$ parametrization, not all combinations of $\alpha \in\left[0,180^{\circ}\right]$ and $\beta \in\left[0,180^{\circ}\right]$ are acceptable. The acceptable limits for $\alpha$, when $\beta=\beta_{0}$, and the limits of $\beta$, when $\alpha=\alpha_{0}$, are independent of the value of the rotation of the $x-y$ frame and may easily be derived from the limitations imposed by (28) and (29). These limits are given in

$$
\text { If } \alpha=\alpha_{0} \text { then }\left\{\begin{array}{c}
90^{\circ}-\alpha_{0} \leq \beta \leq 90^{\circ}+\alpha_{0} \\
\text { for } \alpha_{0} \leq 90^{\circ} \\
\alpha_{0}-90^{\circ} \leq \beta \leq 270^{\circ}-\alpha_{0} \\
\text { for } 90^{\circ}<\alpha_{0} \leq 180^{\circ}
\end{array}\right.
$$

and

$$
\text { If } \beta=\beta_{0} \text { then }\left\{\begin{array}{l}
90^{\circ}-\beta_{0} \leq \alpha \leq 90^{\circ}+\beta_{0} \\
\text { for } \beta_{0} \leq 90^{\circ} \\
\beta_{0}-90^{\circ} \leq \alpha \leq 270^{\circ}-\beta_{0} \\
\text { for } 90^{\circ}<\beta_{0} \leq 180^{\circ} .
\end{array}\right.
$$


Based on the above discussion, and by keeping the framerotation angle $\Theta$ constant (with value $\Theta_{0}$ ), two new families of parameter curves may now be defined

$\{$ the family of $\alpha$-parameter curves (or simply $\alpha$-curves)

$\{$ the family of $\beta$-parameter curves (or simply $\beta$-curves).

Thus, a curve belonging to the family of $\alpha$-curves is defined as the locus of all manifold vectors over the whole $\alpha$ parameter space $\Omega_{\alpha} \equiv\left[\alpha_{\min }, \alpha_{\max }\right]$ at a particular $\beta_{0}$

$$
\text { i.e., } \underline{\hat{\mathrm{a}}}\left(\alpha \mid \beta_{0}, \Theta_{0}\right)=\left.\underline{\hat{\mathrm{a}}}(\alpha, \beta)\right|_{\forall \alpha} ^{\beta=\beta_{0}, \Theta=\Theta_{0}}
$$

with

$$
\alpha_{\min } \quad \text { and } \alpha_{\max } \text { given by (31) }
$$

whereas, in a similar fashion, the family of $\beta$-curves can be defined, with each family covering and representing the whole of the manifold surface of a planar array.

The most important point, however, is that both these families of curves have the property of being complex hyperhelices embedded in $C^{N}$, as it can be seen from (25), which, for instance, for an $\alpha$-curve of constant $\beta\left(\beta=\beta_{0}\right)$ and constant $\Theta\left(\Theta=\Theta_{0}\right)$, becomes identical to (2) with

$\underline{r}=\underline{R}\left(\Theta_{0}\right), p=\alpha$ and $\underline{n}=\underline{R}\left(\Theta_{0}+90^{\circ}\right) \cos \beta_{0}=$ constant.

From the above, it is clear that all $\alpha$-curves have the same ELA $R\left(\Theta_{0}\right)$ but different "visible" areas specified by the minimum and maximum permissible values of $\alpha$. The same is true for the $\beta$-curves but with $\underline{R}\left(\Theta_{0}+90^{\circ}\right)$ as their ELA.

$$
\text { Thus }\left\{\begin{array}{l}
\text { ELA of the family of } \alpha \text {-curves: } \underline{R}\left(\Theta_{0}\right) \\
\quad \text { with the parameter space for a specific } \\
\alpha \text {-curve, } \Omega_{a}=\left[\alpha_{\min }, \alpha_{\max }\right] \\
\text { ELA of the family of } \beta \text {-curves: } \underline{R}\left(\Theta_{0}+90^{\circ}\right) \\
\quad \text { with the parameter space for a specific } \\
\beta \text {-curve, } \Omega_{\beta}=\left[\beta_{\min }, \beta_{\max }\right] .
\end{array}\right.
$$

The above implies that all $\alpha$-curves, for a given frame rotation angle $\Theta_{0}$, are

- identical in shape, having the same sets of curvatures (same Cartan matrix);

- parallel to each other (different displacement values $\underline{n}=$ $\left.\underline{R}\left(\Theta_{0}+90^{\circ}\right) \cos \beta_{0}\right)$

- orthogonal to $\beta$-curves. Note that $\alpha$-curves are the same as $\beta$-curves for rotation $\Theta_{0}+90^{\circ}$.

However, by estimating the rate of change of the arc length of an $\alpha$-curve $\underline{\mathrm{a}}\left(\alpha, \beta_{0}\right)$ for a given rotation $\Theta_{0}$

$$
\text { i.e., } \frac{\partial s}{\partial \alpha}(\alpha)=\pi\left\|\underline{R}\left(\Theta_{0}\right)\right\| \sin \alpha, \quad \forall \alpha \in\left[\alpha_{\min }, \alpha_{\max }\right]
$$

and then integrating (33) over its limits $\alpha_{\min }, \alpha_{\max }$, the length of this $\alpha$-curve can be found as

$$
l_{m_{\alpha \mid \beta_{0}}}=2 \pi\left\|\underline{R}\left(\Theta_{0}\right)\right\| \sin \beta_{0}
$$

which indicates that $\alpha$-curves, although identical in shape, have different lengths, with the $\alpha$-curve associated with $\beta_{0}=$ $90^{\circ}$ having the maximum length and their lengths gradually reducing as $\beta_{0}$ increases from 90 to $180^{\circ}$ or decreases from 90 to $0^{\circ}$.
Based on the above discussion, the ambiguous generator sets of arc lengths of the $\alpha$-curve of $\beta_{0}=90^{\circ}$, i.e., $\underline{\mathrm{a}}\left(\alpha \mid 90^{\circ}, \Theta_{0}\right)$ $\forall \alpha$, can be estimated by partitioning this curve according to the elements (arc lengths) of the following vector [see (8) - uniform partition]:

$$
s_{i, j}=\left[0, \frac{l_{m_{\alpha \mid 90^{\circ}}}}{\left|r_{i}-r_{j}\right|}, 2 \frac{l_{m_{\alpha \mid 90^{\circ}}}}{\left|r_{i}-r_{j}\right|}, \cdots, c \frac{l_{m_{\alpha \mid 90 \circ}}}{\left|r_{i}-r_{j}\right|}\right]^{T}
$$

where $r_{i}, r_{j}$ denote the locations of the $i$ th and $j$ th sensors of the ELA $\underline{R}\left(\Theta_{0}\right)$, as well as, if the array is symmetric, according to the roots of (9) (nonuniform partition).

Now, let us assume that all ambiguous generator sets of arc lengths of the $\alpha$-curve with $\beta_{0}=90^{\circ}$ have been estimated, both of the uniform and nonuniform class (if these exist) and then consider another $\alpha$-curve of $\beta_{0}$ different to $90^{\circ}$. For this second curve, let $s_{\min }$ and $s_{\max }$ be the arc lengths corresponding to the parameter angles $\alpha_{\min }$ and $\alpha_{\max }$ [given by (32)], respectively. This curve has ambiguous sets but not ambiguous generator sets since $s_{\min } \neq 0$ for any $\beta \neq 90^{\circ}$, which implies that the first condition of the definition of the ambiguous generator set is not satisfied. Let us define the ambiguous sets with their first element equal to $s_{\min }$ as the first permissible ambiguous sets. These can be found by rotating all the ambiguous generator sets of the $\alpha$-curve with $\beta_{0}=90^{\circ}$ by $s_{\min }[9$, Th. 1], subject to the condition, of course, that the maximum element of each set cannot exceed $s_{\max }$. Thus, it is clear that the ambiguous generator sets for the whole family of $\alpha$-curves, for a given frame rotation $\Theta_{0}$, can be provided by examining only the $\alpha$-curve of $\beta_{0}=90^{\circ}$. The ambiguities of any other $\alpha$-curve can be generated by a simple rotation [9, Th. 1] of those ambiguous generator sets.

For the array of (19) and for $\Theta_{0}=5^{\circ}$, Fig. 10 shows the ambiguous generator sets of the $\alpha$-curve with $\beta_{0}=90^{\circ}$ as well as the ambiguous sets for variable $\beta_{0}$, which can be produced from the generator sets by adding to their elements the corresponding $s_{\min }$.

Note that the $\alpha$ cone angle parameter, corresponding to the arc lengths $s_{k}$ of the $\alpha$-curve, can be calculated by

$$
\alpha_{k}=\cos ^{-1}\left(1-\frac{s_{k}+\pi\left\|\underline{R}\left(\Theta_{0}\right)\right\|\left(1-\sin \beta_{0}\right)}{\pi\left\|\underline{R}\left(\Theta_{0}\right)\right\|}\right) .
$$

Now, by varying $\Theta_{0}$ over its parameter space $\Omega_{\Theta}=$ $\left[0^{\circ}, 180^{\circ}\right)$, we will get the set of ambiguous generator lines of the whole family of $\alpha$-curves to which the elements of the ambiguous generator sets belong. However, if the set of ambiguous generator lines of the family of $\phi$-curves has been found, then we can also find the set of ambiguous generator lines of the $\alpha$-curves by making the following observation. Although $\hat{\theta}_{0}$ denotes the composite azimuth associated with a $\phi$-curve and $\Theta_{0}$ denotes the frame rotation associated with a family of $\alpha$-curves, that is $\hat{\theta}_{0}$ and $\Theta_{0}$ are totally different parameters, for $\hat{\theta}_{0}=\Theta_{0}$, the corresponding ELA's are identical with their associated hyperhelices having the same length [see (16) and (34) with $\beta_{0}=90^{\circ}$ ]. This implies that their associated ambiguous generator sets of arc lengths are numerically identical. This can be seen for the array of (19), 


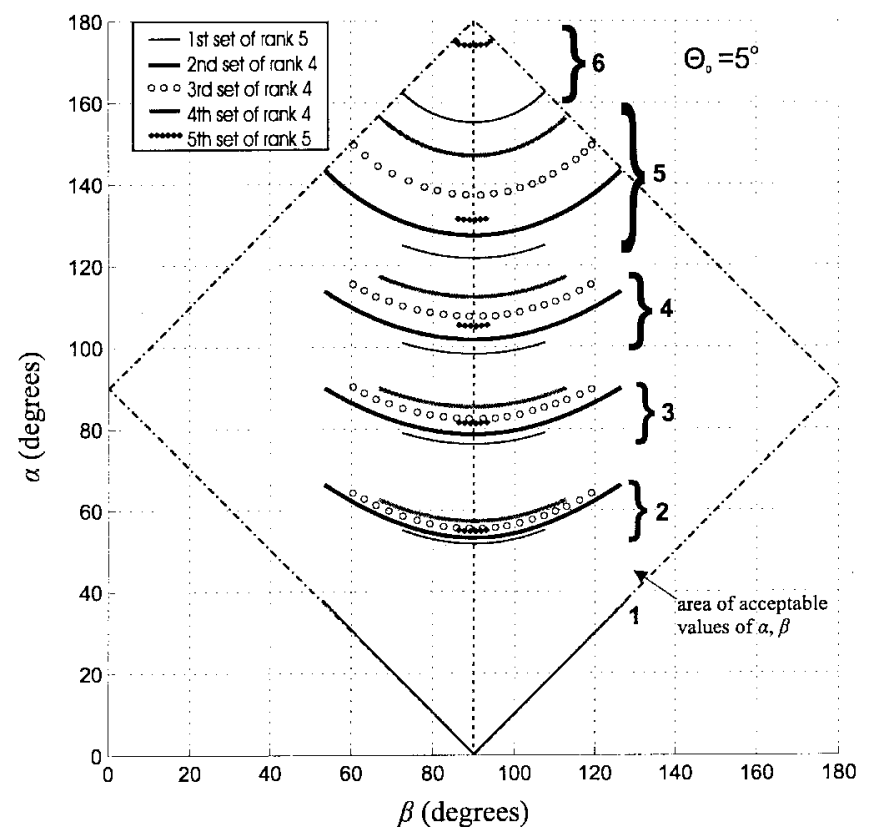

Fig. 10. Ambiguous generator lines for $\Theta_{0}=5^{\circ}$ for the $\alpha$-curves of the planar array given by (19).

which for the family of $\alpha$-curves with $\Theta_{0}=5^{\circ}$, gives the same ambiguous generator sets (in arc lengths) as those found in Section III by examining the $\phi$-curve for $\hat{\theta}_{0}=5^{\circ}$ and are given by the rows of the matrix $\underline{\Sigma}$ of (20).

This implies that, for variable $\overline{\overline{\Theta_{0}}}$ in the region $0-180^{\circ}$ and for the array of (19), the ambiguous generator lines of all families of $\alpha$-curves are numerically, in arc lengths, equal to those of the family of $\phi$-curves. Thus, for the array of (19), Figs. 2-8 not only represent the set of ambiguous generator lines of the $\phi$-curves but also of the $\alpha$ and $\beta$-curves by generalizing the polar axes to $(q, s)$, where

for the composite $\phi$-curves $\begin{cases}q=\hat{\theta}_{0}, & \text { in degrees } \\ s=\phi_{c}, & \text { in arc lengths }\end{cases}$

for the $\alpha$-curves $\begin{cases}q=\Theta_{0}, & \text { in degrees } \\ s=\alpha, & \text { in arc lengths }\end{cases}$

for the $\beta$-curves $\left\{\begin{array}{ll}q=\Theta_{0}+90^{\circ}, & \text { in degrees } \\ s=\beta, & \text { in arc lengths }\end{array}\right.$.

A few other points must also be emphasized before concluding this section. First, from (27), we can derive

$$
\underline{R}(\Theta)=-\underline{R}\left(\Theta+180^{\circ}\right)
$$

which implies that for a given rotation $\Theta_{0}$ of the $x-y$ frame and a given $\beta_{0}$, the resulting $\alpha$-curve $\left.\underline{\hat{a}}\left(\alpha, \beta_{0}, \Theta_{0}\right)\right|_{\forall \alpha} ^{\beta=\beta_{0}}$ has the same length as the $\alpha$-curve $\left.\underline{\hat{a}}\left(\alpha, \beta_{0}, \Theta_{0}+180^{\circ}\right)\right|_{\forall \alpha} ^{\beta=\beta_{0}}$ resulting from rotation $\Theta_{0}+180^{\circ}$. This is the reason that only frame rotations $\Theta_{0}$ in the region $\left[0^{\circ}, 180^{\circ}\right]$ have been considered. Second, as already mentioned, the roles of $\alpha$ and $\beta$ can be interchanged by simply replacing the rotation $\Theta_{0}$ of the $x-y$ frame with $\Theta_{0}+90^{\circ}$, and therefore, the $\beta$-curves are effectively transposed versions of the $\alpha$-curves. This means that the $\alpha$ curves for some rotation $\Theta_{0}$ are the same with the $\beta$-curves for rotation $\Theta_{0}+90^{\circ}$. This is the reason the $\beta$-curves can be neglected throughout this analysis, and only the $\alpha$-curves are considered.

\section{Discussion on Special Cases of Planar Arrays}

As it was noted in Fig. 4, for certain values of $\hat{\theta}_{0}$ or $\Theta_{0}$, there are some ambiguous generator sets of considerably lower rank than those belonging to the sets of the ambiguous generator lines that are presented in Fig. 4 as singular points (squares). These ambiguous generator sets, known as "discrete" ambiguous generator sets, exist when the array geometry satisfy one of the following two conditions for some values of $\hat{\theta}_{0}$ or $\Theta_{0}$

Condition 1: The corresponding ELA's have two or more co-located sensors.

Condition 2: Two or more intersensor spacings of the corresponding ELA's are integer multiples of each other.

The reason for the existence of these lower rank ambiguities can easily be deduced from the definition of the ambiguous generator set and, especially, its third condition. More analytically, it can easily be derived from [9, proof of Th. 1] that if two or more intersensor spacings of an ELA are integer multiples of each other (which is the case for either of the above conditions) and if $\underline{s}$ is an ambiguous generator set associated with the smallest of these intersensor spacings, then the manifold matrix $\mathbb{A}(\underline{s})$ may have more than two identical rows. This implies that its rank will be smaller than $N-1$.

Note that an ELA has two or more co-located sensors when for one or more different pairs of sensors, the parameter $q$ satisfies

$$
\tan q=-\frac{r_{x i}-r_{x j}}{r_{y i}-r_{y j}}, \quad i \neq j
$$

where $q=\hat{\theta}_{0}$ or $q=\Theta_{\mathrm{o}}, q \in\left[0,180^{\circ}\right)$ and $\left(r_{x i}, r_{y i}\right)$, $\left(r_{x j}, r_{y j}\right)$ represent the locations of the $i$ th and $j$ th sensors. It can be proven that for a given array geometry, the maximum number $n_{\max }$ of ELA's with co-located sensors is given by

$$
n_{\max }(\underline{\underline{\mathbf{r}}})=\frac{N^{2}-N}{2}-2 \delta\left(\begin{array}{c}
m \\
2
\end{array}\right)-2 m \epsilon
$$

where $m$ is the number of symmetric pairs with respect to the origin in the planar array

$$
\epsilon= \begin{cases}1, & \text { if the array has a sensor at the origin } \\
0, & \text { otherwise }\end{cases}
$$

and

$$
\delta= \begin{cases}1, & \text { if } m>1 \\ 0, & \text { otherwise }\end{cases}
$$

For instance, in Fig. 4, for $q=45^{\circ}$ or $q=135^{\circ}$, we have seen that there is a "discrete" ambiguous generator set. By examining the ELA for these two values of $q$, it can be seen that the second condition is satisfied. Furthermore, the first condition is also satisfied at $q=0^{\circ}$, where, although not shown in Figs. 3-8, there are five "discrete" ambiguous generator sets of rank 3 and 11 of rank 2. To better illustrate the condition of co-located sensors, consider, for instance, a uniform circular array (UCA) with eight elements and 1.5 halfwavelengths intersensor spacing. Equation (39) will give us 


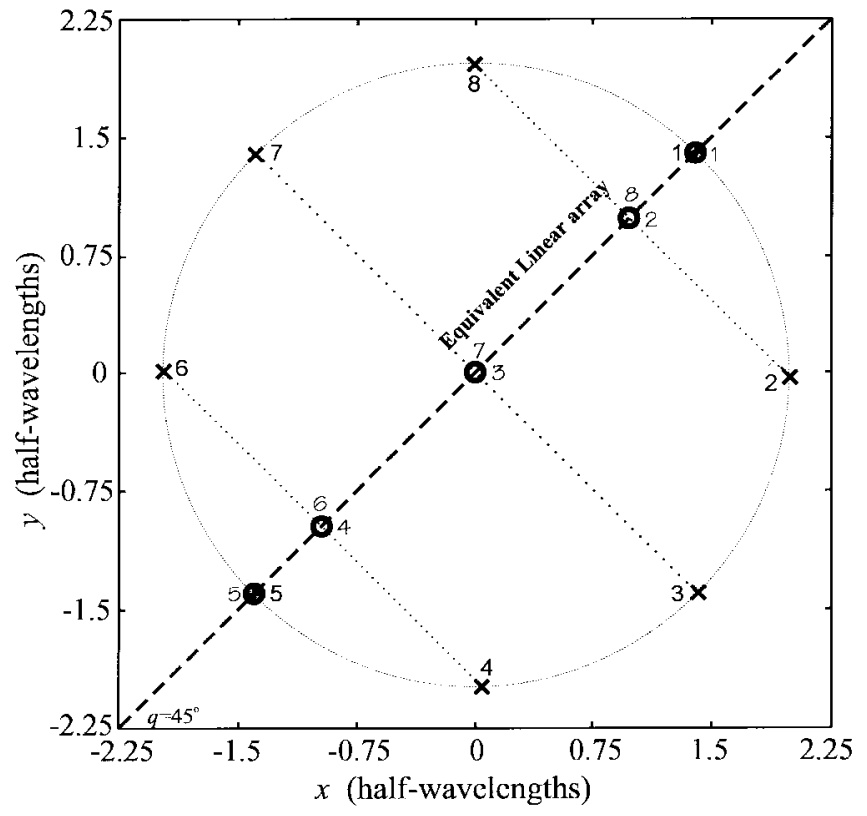

Fig. 11. Planar array configuration and corresponding ELA for $q=45^{\circ}$ for a UCA of 1.5 half-wavelengths intersensor spacing. $\mathbf{x}$ represents the sensors locations, and o represents the locations of the ELA.

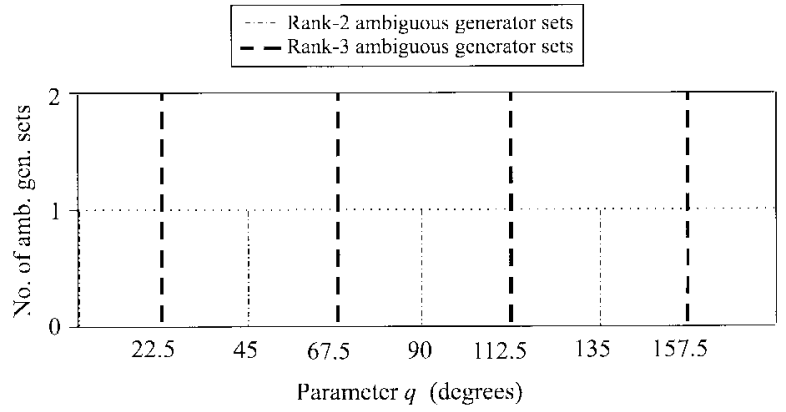

Fig. 12. Number of ambiguous generator sets, grouped by rank, with respect to $q$ for a UCA with 1.5 half-wavelengths intersensor spacings.

that the maximum number of ELA's with co-located sensors is $n_{\max }=8$. Indeed, the array has eight ELA's with colocated sensors corresponding to $q=0,22.5,45,67.5,90$, $112.5,135$, and $157.5^{\circ}$. In Fig. 11, the ELA for $q=45^{\circ}$ is presented (with the circles) and has only one ambiguous generator set of rank two, as shown in Fig. 12. Furthermore, in this figure, the number of ambiguous generator sets over the whole parameter space are shown grouped by rank. Note that for this array, only "discrete" ambiguous generator sets exist at the previously mentioned values of $q$ and not sets of ambiguous generator lines.

By increasing the intersensor spacing further to 2.5 , not only is the number of "discrete" ambiguous generator sets increased, but there is also one set of ambiguous generator lines of rank- 6 covering the areas of $\pm 6^{\circ}$ around the positions of the "discrete" ambiguous generator sets, as can be seen in Fig. 13. In addition, new "discrete" ambiguous generator sets of rank-4 are shown in the same figure. Finally, in Fig. 14, the histogram for intersensor spacing equal to 3 is shown. It is clear from this figure that the number of "discrete" ambiguous generator sets is dramatically increased while the

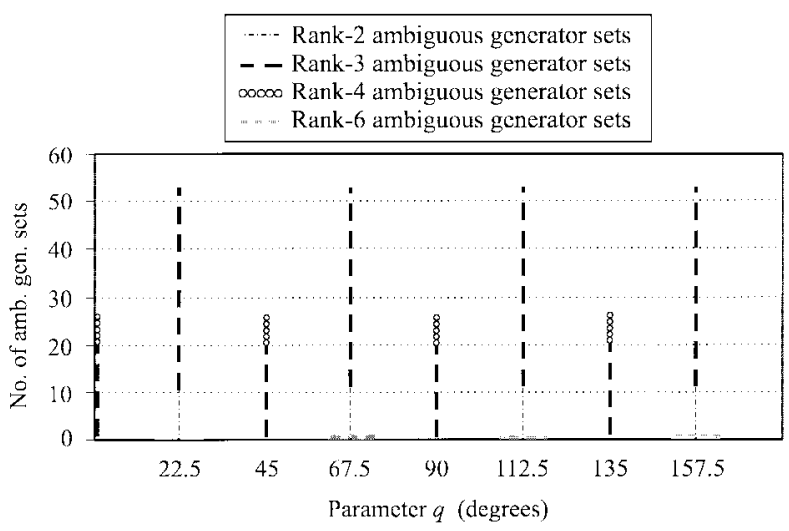

Fig. 13. Number of ambiguous generator sets, grouped by rank, with respect to $q$ for a UCA with 2.5 half-wavelengths intersensor spacings.

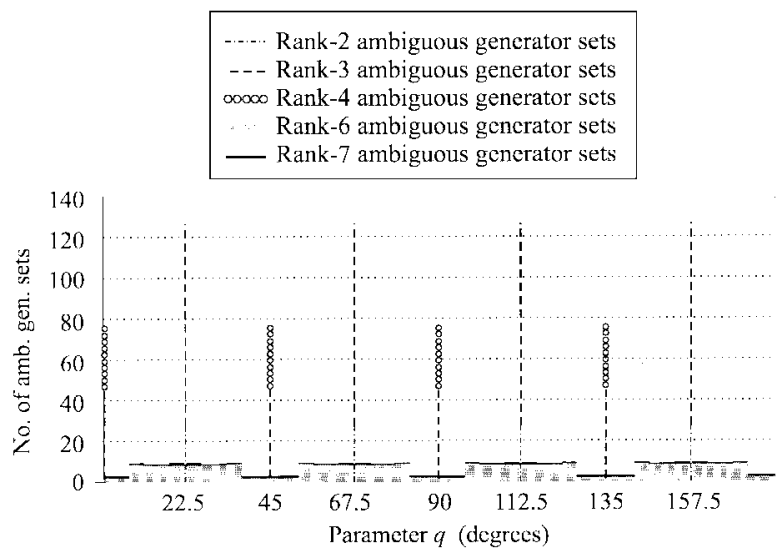

Fig. 14. Number of ambiguous generator sets, grouped by rank, with respect to $q$ for a UCA with three half-wavelengths intersensor spacings.

sets of ambiguous generator lines cover the whole parameter space $\Omega_{p}=\left[0^{\circ}, 180^{\circ}\right)$, with their number increased to ten in some parts and to three in some other parts of $\Omega_{p}$. In addition, some new ambiguous generator lines of rank-7 also exist.

The above example is a characteristic one that illustrates the importance of the array geometry in the ambiguity problem and in the presence of lower rank ambiguities. Note that all symmetric array geometries (grid, $X, Y$ shaped arrays etc.) have similar properties to those of the uniform circular array.

\section{CONCLuSions}

In this paper, a method for estimating ambiguous generator sets for planar arrays was proposed. This method was based on the concept of hyperhelices and the special properties of hyperhelical curves. These properties make it possible to estimate ambiguities for constant azimuth and different elevations and for different azimuths and different elevations based on both uniform and nonuniform partitions of the array manifold. Finally, some special cases of low-rank ambiguous generator sets were discussed.

\section{REFERENCES}

[1] H. R. Karimi and A. Manikas, "Manifold of a planar array and its effects on the accuracy of direction-finding systems," Proc. Inst. Elect. Eng., Radar, Sonar Navigation Syst., vol. 143, no. 6, pp. 349-357, Dec. 1996. 
[2] M. Wax and I. Ziskind, "On unique localization of multiple sources by passive sensor arrays," IEEE Trans. Acoust., Speech, Signal Processing, vol. 37, pp. 996-1000, July 1989.

[3] A. Nehorai, D. Starer, and P. Stoica, "Direction-of-arrival estimation in applications with multipath and few snapshots," Circuits Syst. Signal Process., vol. 10, no. 3, pp. 327-342, 1991.

[4] K. C. Ho, K. C. Tan, and W. Ser, "An investigation on number of signals whose direction-of-arrival are uniquely determinable with an electromagnetic vector sensor," Signal Process., vol. 47, no. 1, pp. 41-54, Nov. 1995.

[5] B. Hochwald and A. Nehorai, "Identifiability in array processing models with vector-sensor applications," IEEE Trans. Signal Processing, vol. 44, pp. 83-95, Jan. 1996.

[6] K. C. Tan and Z. Goh, "A detailed derivation of arrays free of higher rank ambiguities," IEEE Trans. Signal Processing, vol. 44, pp. 351-359, Feb. 1996.

[7] T. H. Lo and S. L. Marple, "Observability conditions for multiple signal direction finding and array sensor localization," IEEE Trans. Signal Processing, vol. 40, pp. 2641-2650, Nov. 1992

[8] K. C. Tan, E. C. Tan, and S. S. Goh, "A study of the rank-ambiguity issues in direction-of-arrival estimation," IEEE Trans. Signal Processing, vol. 44, pp. 880-887, Apr. 1996.

[9] A. Manikas and C. Proukakis, "Modeling and estimation of ambiguities in linear arrays," IEEE Trans. Signal Processing, vol. 46, pp. 2166-2179, Aug. 1998.

[10] C. Proukakis and A. Manikas, "Study of ambiguities of linear arrays," in Proc. IEEE ICASSP, 1994, vol. IV, pp. 549-552.

[11] M. Lipschutz, Differential Geometry. New York: McGraw-Hill, 1969.

[12] H. Guggenheimer, Differential Geometry. New York: McGraw-Hill, 1969.

[13] I. Dacos and A. Manikas, "Estimating the manifold parameters of onedimensional arrays of sensors," J. Franklin Inst. Eng. Appl. Math., vol. 332B, no. 3, pp. 307-332, July 1995.

[14] http://skynet.ee.ic.ac.uk/wwwam/software/arraycommdownload.html, MatLab function "hyperhelix.m."

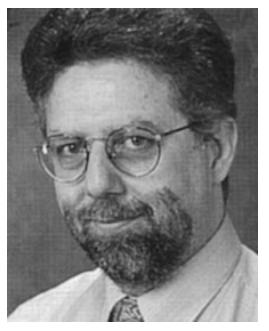

Athanassios Manikas (M'88) received the Ph.D and D.I.C. degrees from Imperial College, University of London, London, U.K., in 1988.

He was appointed a Lecturer at Imperial College in 1988. He is now a Reader in digital communications with the Department of Electrical and Electronic Engineering, Imperial College of Science, Technology, and Medicine, London. He has published extensive sets of journal and conference papers relating to his research interests, which are in the general area of communication and signal processing, where he has developed a wide and deep interest in the topic of super-resolution array processing and the application of array theory to communication systems. He has been involved in array manifold investigations using differential geometry, super-resolution beamformers, direction finding, "near" and "far" field array processing, performance bounds in array systems, array uncertainties and ambiguities, array design, array signal abnormalities and quality factors, higher order signal subspace techniques, $\mathrm{H}$-inf-type robust array processing, robust array receivers, and integrated wireless array communication networks based on CDMA, GSM-like, etc., platforms. Furthermore, he is the Organizer of the Advanced Communications and Signal Processing Laboratory at Imperial College and, over the last nine years, has held a number of important research consultancies for the EU, industry, and government organizations. He is currently the Deputy Head of the Communications and Signal Processing Research Group at Imperial College.

Dr. Manikas is a member of IEICE, a corporate member of IEE, and a Chartered Electrical Engineer.

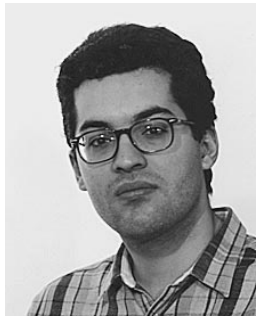

Christos Proukakis received the Diploma in electrical engineering from the National University of Athens, Athens, Greece, in 1990, the M.Sc. degree in communications and signal processing in 1991, and the Ph.D. and D.I.C. degrees in electrical engineering in 1997, all from Imperial College of Science, Technology, and Medicine, University of London, London, U.K.

His research interests are in the areas of sensor array signal processing and super-resolution spatiotemporal spectral estimation, as well as the general area of digital communications.

Dr. Proukakis was awarded the Frangoulis Memorial studentship in 1992 and was a scholar of the Onassis Foundation from 1993 to 1996 . He is a member of the Technical Chamber of Greece.

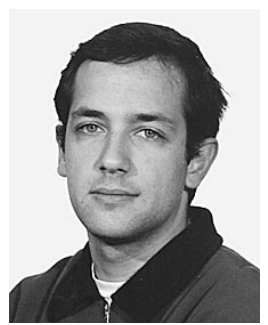

Vasileios Lefkaditis received the B.Sc. degree in physics from the University of Athens, Athens, Greece, in 1996.

Since October 1996, he has been a Research Student at the Communications and Signal Processing Research Group, Department of Electrical and Electronic Engineering, Imperial College of Science, Technology and Medicine, University of London, London, U.K. His research interests are in the areas of array processing, harmonic retrieval problems, engineering applications of differential geometry, estimation theory, and the general area of digital communications.

Mr. Lefkaditis has been a scholar of the Greek State Scholarships Foundation (IKY) since October 1997 and a student member of IEE. 